\title{
I mpacto del informe de la Comisión sobre Determinantes Sociales de la Salud cuatro años después
}

\author{
I mpact of the Report of the WHO Commission on Social \\ Determinants of Health four years after publication
}

\author{
Dr. C. J oan Benach de Rovira,' Dr. C. Carles Muntaner Bonet,"' Dr. C. Gemma \\ Tarafa,' Dr. C. Montse Vergara' \\ I GREDS/EMCONET, Universitat Pompeu Fabra. Barcelona, España. \\ II Universidad de Toronto. Toronto, Canadá
}

\section{RESUMEN}

Tras más de tres años de trabajo, la Comisión de Determinantes Sociales de la Salud de la Organización Mundial de la Salud, presentó a finales de agosto de 2008 su informe final traducido en su versión castellana como "Subsanar las desigualdades en una generación: alcanzar la equidad sanitaria actuando sobre los determinantes sociales de la salud". El informe, muy probablemente uno de los textos de mayor interés en el campo de la salud pública de las últimas décadas, no ha dejado indiferente. Ha recibido notables alabanzas, desaforadas críticas y algunas -las menos hasta el momento- críticas con un carácter más equilibrado y razonable. El objetivo de este texto es apreciar críticamente dicho informe y valorar su impacto cuatro años después de su publicación. Para ello, en primer lugar, se revisa el origen y objetivos de la Comisión resumiendo la visión y difusión del informe; segundo, se valoran las características y contenidos del informe desde distintos puntos de vista ideológicos y políticos: neoliberal y conservador, desde la epidemiología social y desde una visión radical de salud pública; y tercero, se evalua el impacto global del informe cuatro años después de su publicación. El texto concluye con algunas reflexiones finales.

Palabras clave: desigualdades, determinantes sociales, OMS, revisión, Comisión sobre Determinantes Sociales de la Salud (CDSS).

\section{ABSTRACT}

After more than three years of work, the WHO Commission on Social Determinants of Health launched its final Report "Closing the gap in a generation: Health equity 
through action on the social determinants of health" at the end of August 2008. This report, which is probably one of the most interesting texts in the field of public health in recent decades, has attracted a lot of attention. It has been the object of remarkable praises, of ardent criticism, and to lesser extent, of more balanced and reasonable critiques. The aim of this paper was to critically evaluate the report and to assess its impact four years after its publication. Firstly, the origins and objectives of the Commission was reviewed, outlining the vision and dissemination of the report; secondly, the characteristics and contents of the report were assessed from different ideological and political points of view including neoliberal, conservative, social epidemiology and radical view of public health; and finally, the overall impact of the Report four years after publication was evaluated. The paper concluded with some final reflections.

Key words: health inequalities, social determinants, World Health Organization, review, Commission on Social Determinants of Health (CSDH).

\section{NTRODUCCI ÓN}

La Comisión sobre Determinantes Sociales de la Salud de la Organización Mundial de la Salud (OMS) presentó a finales de agosto de 2008 el Informe final de la Comisión (IC) titulado "Subsanar las desigualdades en una generación: alcanzar la equidad sanitaria actuando sobre los determinantes sociales de la salud". ${ }^{1}$ El informe, muy probablemente uno de los textos de salud pública más influyentes e importantes de las últimas décadas, no ha dejado indiferente y ha recibido notables alabanzas, desaforadas opiniones y algunos críticas con un carácter más equilibrado y razonable. El objetivo de este artículo es realizar una valoración crítica global de dicho informe así como de su impacto general cuatro años después de su publicación.

\section{LA COMI SI ÓN DE DETERMI NANTES SOCI ALES DE LA SALUD (2004-2008)}

\section{Origen, objetivos, visión y difusión}

A raíz de la $57^{a}$ Asamblea Mundial de la Salud de la OMS, donde se analizaron los principales problemas de salud de la humanidad el entonces director general de la OMS, el Coreano Lee Jong-wook, anunció la creación de una Comisión sobre Determinantes Sociales de la Salud (CDSS), para resumir la información científica sobre cómo las relaciones de empleo, la globalización, los servicios sanitarios, la exclusión social, el género, el ambiente urbano, o el desarrollo infantil, producen desigualdades en salud en el mundo entero; así como generar recomendaciones políticas para su reducción.

El número de personas que intervinieron en la Comisión fue muy numeroso: científicos, expertos de la OMS y otros organismos de la Organización de Naciones Unidas (ONU), políticos y, algo poco común en otras comisiones, también miembros de la llamada sociedad civil y de organizaciones no gubernamentales (ONGs). El director de la Comisión y principal responsable del IC fue el epidemiólogo británico Michael Marmot acompañado por su equipo de la University College London (UCL) en 
Londres y un amplio grupo de comisionados del mundo de la política y la ciencia como el Nobel de Economia Amartya Sen. Además, en todo el proceso jugaron un papel muy importante las llamadas "Redes de conocimiento", que recibieron el encargo de resumir el conocimiento científico disponible en áreas específicas. ${ }^{2}$

Fruto de la amplia diversidad de comisionados, grupos sociales, investigadores, gobiernos y ONGs, se realizó, en general, un IC de carácter innovador donde se plantean temas relativos a la justicia social, el empleo justo, la financiación de los países pobres, la salud en todas las políticas, la regulación del mercado, y la necesidad de políticas públicas. ${ }^{3}$

El IC se presentó el 28 de agosto de 2008 junto a Margaret Chan (la nueva directora de la OMS). Los esfuerzos de difusión a través de la colaboración con las Naciones Unidas en diversas iniciativas globales, las reuniones con ministros de salud en Bamako en noviembre de 2008, o la discusión sobre la generación de un "movimiento" para eliminar las desigualdades en salud a través de la participación de las poblaciones afectadas, han sido importantes objetivos derivados de la publicación del informe. Con todo ello, y a pesar del relativamente bajo perfil inicialmente adquirido por el Informe en el seno de la OMS (por ejemplo, con un lanzamiento modesto), el informe fue capaz de captar la atención dentro y fuera del campo de la salud pública.

\section{El contenido del informe de la Comisión sobre Determinantes Sociales de la Salud}

La valoración más importante del IC relativa al origen de los determinantes sociales (DS) tiene que ver con las llamadas "causas de las causas". Es la "combinación tóxica" de factores sociales la que daña la salud de las clases sociales más pobres, con menos recursos y en peor situación social. Las "vías" o caminos causales pueden ser distintos según los individuos y grupos sociales, pero son los individuos en condiciones más desfavorables los más explotados u oprimidos, son quienes tienen una mayor probabilidad de alimentarse inadecuadamente, de fumar y beber alcohol en exceso con mayor frecuencia debido a su estrés, y de tener servicios sanitarios menos completos y de menor calidad. Además, el IC contiene también una amplia lista de recomendaciones y prioridades que tienen que ver con mejorar las condiciones de vida cotidianas, medir y comprender los problemas y evaluar el impacto de las intervenciones a realizar, y la más innovadora, luchar contra la distribución desigual del poder y los recursos.

\section{VALORACI ONES Y CRÍ TI CAS AL I NFORME DE LA COMI SI ÓN SOBRE DETERMI NANTES SOCI ALES DE LA SALUD}

La publicación final del IC provocó una amplia gama de reacciones. A continuación, se revisan los principales tipos de críticas: la visión muy negativa realizada por fuerzas neoliberales, la visión positiva realizada por epidemiólogos sociales, y finalmente, la visión positiva pero crítica constructiva desde el ámbito de la salud pública, que se presenta de forma más exhaustiva.

\section{La visión catastrofista y descalificante neoliberal}

Desde el punto de vista conservador planteado por revistas neoliberales, laboratorios de ideas (think tanks) conservadores o el mundo académico, se ha realizado una 
valoración negativa o muy negativa del informe, con una crítica destructiva del mismo. Algunas de las principales respuestas realizadas pueden resumirse en cinco puntos principales. Primero, la descalificación ideológica, política e incluso científica. La revista de ideología neoliberal The Economist, ${ }^{4}$ o el Instituto Fraser ${ }^{5}$ y sus ideólogos, ${ }^{6}$ por ejemplo, han señalado que el informe puede estar en lo cierto al tratar el conjunto de causas que determinan la salud de las personas, pero que es políticamente ingenuo y se equivoca al no tener en cuenta el papel fundamental del crecimiento económico. ${ }^{4}$ Por su parte, algunos autores han centrado sus críticas en la descalificación académica. ${ }^{7}$ Segundo, se reprueba que el IC no valore que el crecimiento económico y la globalización han tenido también importantes efectos positivos para la salud y bienestar de la población..$^{5,7}$ Tercero, se desacredita la conclusión sobre el rol beneficioso de la protección social, y la regulación del empleo y el comercio. ${ }^{5}$ Cuarto, se reprocha que no se plantee la importancia de la responsabilidad personal sobre la salud o el debate sobre las libertades individuales. ${ }^{7}$ Y quinto, se descalifica por no plantear donde invertir recursos médicos escasos. ${ }^{4}$

\section{La visión complaciente de la epidemiología social}

La respuesta al IC en los artículos realizados desde la visión de la epidemiología social, y propuesto por investigadores asociados al campo de las desigualdades en salud ha sido en general positiva o muy positiva. La visión "progresista" favorable entre diversos epidemiólogos sociales puede resumirse en los siguientes puntos. En primer lugar, se valora positivamente el hecho de que una agencia mundial como la OMS trate el tema de la desigualdad en salud. ${ }^{8,9}$ Segundo, se reconoce que el IC presenta gran cantidad de información, siendo por vez primera un informe "global" sobre el tema. ${ }^{8,10}$ Tercero, se plantea el tema de la política y de las relaciones políticas sobre la salud, ${ }^{8}$ y la inclusión en el proceso del papel de la sociedad civil, también valorado en prestigiosas revistas científicas como The Lancet. ${ }^{11}$ Cuarto, se menciona explícitamente y de forma reiterada el "empoderamiento" y la "participación" como factores políticos de gran importancia. ${ }^{8}$ Y finalmente, se valora el hecho de que el crecimiento económico no es suficiente para generar equidad. ${ }^{8}$

\section{La visión radical de la salud pública}

Desde el punto de vista de la salud pública, se valora favorablemente el exhaustivo trabajo para reunir evidencias sobre los determinantes y las desigualdades, hacer visible las relaciones entre desigualdades sociales y desigualdades en salud, la generación de recomendaciones que contribuyan a la reducción de las desigualdades en salud o, por citar solo algunas, su insistencia en realizar políticas que garanticen servicios esenciales con independencia de la capacidad de pago de las personas. Sin embargo, también se deben enfatizar algunas importantes debilidades e insuficiencias que a continuación se detallan. En primer lugar, el IC no es especialmente novedoso, ni aporta ideas muy originales, tal y como apuntan ciertos análisis procedentes del ámbito de la medicina social o la salud colectiva latinoamericana ${ }^{12} \mathrm{o}$ canadiense. ${ }^{13}$ Segundo, a pesar de su enfoque multidisciplinar, el IC no señala o al menos no plantea con la suficientemente profundidad, el tema relativo a la interrelación "compleja" entre determinantes, causas o factores. ${ }^{12}$ Asismismo, existen discrepancias con la concepción de la salud colectiva latinoamericana, en cuanto a los modelos epistemiológicos y teóricos de la determinación social de la salud y entre los determinantes del cambio social. ${ }^{14}$ Tercero, no se realiza un análisis histórico y geográfico de los fenómenos y problemas tratados. Así, en gran medida los procesos explicados aparecen en forma "asocial" y "ahistórica". ${ }^{12,15}$ Cuarto, como reflejo de las anteriores insuficiencias, se puede observar que determinados temas de salud pública de gran importancia, o bien son insuficientemente tratados, o bien se encuentran prácticamente olvidados. Tres de los "olvidos" que parecen más relevantes y que se 
detallan a continuación hacen referencia a la crisis socioecológica, la guerra y el militarismo, y la clase social. El medio ambiente y la ecología juegan un papel muy relevante en la salud pública y a su vez gran parte de los problemas ambientales derivados de la crisis ecológica global tienen sus orígenes y consecuencias en causas sociales y políticas. Por otra parte, la guerra constituye un determinante de gran relevancia para la salud pública y la creación de desigualdades en salud. No solo por los recursos que implica de inversiones sociales acuciantes, sino por el daño directo e indirecto que promueven. ${ }^{16-19}$ Finalmente, cabe mencionar la falta de uso del concepto de clase social que constituye un mecanismo explícito relacional (propiedad, gestión) que explica cómo las desigualdades económicas se generan y cómo estas pueden afectar a la salud, ${ }^{20}$ en contraposición de las medidas habituales de estratificación social.

Por último, el IC no analiza con la suficiente profundidad los procesos políticos ni de participación social, ${ }^{21,22}$ que a su vez se traduce en una falta de especificidad en las recomendaciones finales del informe. ${ }^{3}$ Tener en cuenta los factores políticos es crucial para explicar los determinantes sociales de la salud (DSS). No se trata únicamente de señalar "las causas de las causas", sino "las causas de las causas de las causas", ${ }^{23}$ cuando se trata de abordar la desigual distribución en el poder político, económico y social, que condiciona las políticas sociales y económicas, lo cual a su vez influye en la salud de las personas.

\section{CUATRO AÑOS DESPUÉS: I MPACTO DEL I NFORME DE LA COMISI ÓN SOBRE DETERMI NANTES SOCI ALES DE LA SALUD}

El IC no es un documento científico original sino un trabajo de revisión y divulgación, cuyo impacto ha de manifestarse en la continuidad del trabajo salubrista en los DSS. Así pues, podemos evaluar el impacto del informe a través del seguimiento que se le ha dado al tema de los DSS en dos marcos instucionales: a. Entre los estados miembros de las Naciones Unidas, el organismo internacional que lo publicó. b. Los distintos países de la ONU en el contexto internacional y también, la continuidad del trabajo en DSS en el seno de la OMS. En el 2008, después de publicar el informe, la Asamblea general de la ONU aprobó una resolución sobre los DSS. Se hicieron concesiones para lograr el consenso (en cuestiones de género y homosexualidad, por ejemplo). Sin embargo, la necesidad de atajar los DSS para subsanar las desigualdades en salud a nivel global quedó firmemente reconocida. Por desgracia, el liderazgo de la OMS, siguiendo la pauta marcada por la muerte del Dr. Lee, siguió sin aprovechar la oportunidad que brindó la publicación del informe. Sin embargo, los países miembros por su parte tomaron nota del informe y mucho de ellos, hasta la fecha, siguieron pujando por una acción concentrada en los DSS.

Cabe destacar aquí el liderazgo de Brasil durante el periodo de Lula. Brasil se ofreció para organizar la conferencia de Río en Octubre de 2011 sobre DSS que sirvió de continuidad al informe, cuya realización ya havia estado planeada por la Comisión. Sin embargo, a pesar de la voluntad brasileña de potenciar la continuidad del IC, la conferencia fue un paso atrás. El documento final no estuvo a la altura del informe, al contrario, parecía desdecirse de la contundencia de aquel. De hecho, parecía como si el liderazgo de la OMS quisiera distanciarse del informe que tan poco había hecho por divulgar en el verano del 2008. Muchos ejemplos reafirman esta impresión. Michael Marmot, el director de la Comisión no participó en el relato final de la declaración de Rio cuyo lenguage, como ya se dijo, fue bastante inconcluso y vago con respecto a la reducción de las DSS. Entre los ponentes hubo pocos representantes del sector civil, aparte del "moderado" People's Health Movement, cuyas opiniones abarcan un espectro político muy amplio. Un ministro griego habló de la necesidad de imponer 
medidas de austeridad ante la crisis, el ministro de salud de los EE. UU. se extendió hablando del sistema privado estadounidense sin tan siquiera mencionar el éxito de los sistemas públicos de sus vecinos cubanos y canadienses, la directora de la OMS evitó hablar del futuro de las DSS, no habló del IC, sino que habló de temas no directamente relacionados, como son las enfermedades crónicas. Todo ello contribuyó a la impresión general de que aunque algunos países siguen interesados en las políticas de los DSS y en el análisis político que de ellos hizo el IC, la OMS se distanciaba de este tema y otros países de peso en la ONU consideraban el tema demasiado político para su gusto.

Por otro lado, la evolución de los DSS en el seno mismo de la OMS no augura tampoco un futuro muy esperanzador. Es cierto que el cambio de liderazgo en la OMS no resultó en la eliminación immediata de las áreas y subdivisiones dedicadas a los DSS. Equidad, Género, Derechos Humanos y DSS han seguido contando con personal y presupuesto durante los años siguientes al IC. Sin embargo, la nueva reforma interna que se anuncia puede eliminar casi por completo la presencia de los DSS de la organización. Por un lado, la reduccion de personal puede dejar a veces con una sola persona a cargo de alguna de estas areas de DSS. Por otro, la estrategia de mainstreaming, aunque a priori, parece indicar que los DSS se incorporarían como parte sustantiva de todas las divisiones, en la práctica pudiera resultar en su desaparición. Es decir, si todos han de hacer DSS, como añadido a sus funciones principales (por ejemplo, las enfermedades transmisibles), es posible que a la hora de la verdad, y sobre todo, no haya fondos y nadie lo haga.

En resumen, el momento de popularidad de que gozaron los DSS a raiz de la publicación del IC en 2008, no se ha visto reforzado por la OMS y otras instituciones de la ONU tal como hubiera sido deseable.

\section{REFLEXI ONES FI NALES}

Conocer, reducir, y eliminar las desigualdades en salud debe constituir un objetivo clave de salud pública por al menos tres razones fundamentales: primero, para hacer visible una grave epidemia sanitaria; segundo, porque las desigualdades en salud son diferencias injustas que se pueden evitar; y tercero, porque las desigualdades en salud constituyen un indicador crucial de justicia social. A pesar de sus limitaciones, el informe de la CDSS constituye un importante documento que coloca las desigualdades sociales en salud en el mapa político global y apunta a soluciones políticas que pueden ayudar a reducir esas desigualdades. Desde este punto de vista, su importancia quizás es comparable a los primeros documentos de la OMS sobre los efectos nocivos del tabaco o la conferencia de Alma-Atá sobre salud primaria.

De forma esquemática, los principales pasos para reducir las desigualdades en salud podrían resumirse así: 1. Difundir el interés y la necesidad de conocer, estudiar y eliminar las desigualdades en salud. 2. Dotar con mayores recursos humanos y económicos a los investigadores de esta materia. 3. Analizar las desigualdades en salud a través de los indicadores y mecanismos sociales más adecuados. 4. Plantear las recomendaciones y prioridades más pertinentes. 5 . Generar el contexto político que cree los recursos humanos y económicos y las fuerzas políticas adecuadas para realizar las intervenciones más urgentes y necesarias. 6 . Poner en práctica y evaluar aquellas intervenciones que efectivamente reduzcan las desigualdades en salud, y especialmente allí donde es más necesario. El informe de la CDSS debe valorarse fundamentalmente en relación con los cuatro primeros pasos. ¿Cuáles pueden haber sido hasta el momento sus logros? En relación con el primer punto, todo parece indicar que se ha alcanzado un cierto éxito, ya que parece estar aumentando el 
interés por el tema así como el número de investigadores y expertos en salud que reconocen la importancia de los determinantes sociales en la generación de la desigualdad en salud. En cuanto al 2, podemos estimar que, aún con las reducciones presupuestarias de la investigación científica existentes bajo la actual crisis económica, no parece haberse reducido ni el interés ni los recursos, al menos en forma relativa, dirigidos al tema. Por lo que hace al punto 3, ya han sido comentados los importantes logros del IC, pero también algunas de sus limitaciones, donde brillan por su ausencia mecanismos explicativos generadores de la desigualdad como, por ejemplo, los modos de dominación neocoloniales, o los mecanismos de explotación, dominación o discriminación de los trabajadores. En relación con el punto 4, cabe decir que las recomendaciones del IC son bastante timoratas, quedándose en un ámbito demasiado genérico del tipo de hay que "mejorar las condiciones de vida", "repartir recursos" o "hacer más estudios."

En otro artículo hemos apuntado algunas de las prioridades relacionadas con la investigación y las recomendaciones que sería necesario desarrollar. ${ }^{24}$ Destaquemos aquí tres de las más importantes: a. Hacer análisis de "soluciones" y no solo de "problemas". b. Mejorar los métodos para evaluar no solo qué intervenciones pueden ser más efectivas y con mayor impacto, sino también por qué, cómo, y en qué contextos pueden reducirse más efectivamente las desigualdades. c. Coordinar e integrar diferentes estrategias y que sean sostenibles a largo plazo.

En relación con los pasos 5 y 6 , aún es demasiado pronto para valorar el papel jugado por la CDSS. Sabemos que ni el conocimiento, ni las propuestas, soportan necesariamente la puesta en práctica de acciones reales ni de acciones que sean efectivas. Las conclusiones y recomendaciones de los informes no toman decisiones. Si el IC de la CDSS ayudará o no al cambio político que sitúe el tema en un primer plano entre los objetivos políticos de los gobiernos afectados, es algo que está por ver, aunque no parece probable. Las recomendaciones del IC no plantean la imprescindible necesidad de hacer frente de forma radical a intereses muy poderosos, y por tanto, cambiar drásticamente la estructura de poder, propiedad y riqueza actuales. En ese sentido, nos parece muy importante ampliar y profundizar los procesos de participación real, especialmente en el ámbito laboral, local y comunitario, que aumenten el poder de las fuerzas políticas que están más en favor de la equidad en salud. Finalmente, el IC no aborda tampoco con la suficiente contundencia y claridad, la necesidad de experimentar propuestas sociales y económicas alternativas al capitalismo, mediante la propuesta de otras formas de producir y de consumir bajo un sistema económico que sea más democrático y respetuoso con la población, el medio ambiente y la equidad. Como en cualquier otro campo, el impacto final que pueda tener dependerá de las distintas posturas ideológicas y, sobre todo, de las correlaciones de fuerzas políticas existentes.

\section{REFERENCI AS BI BLI OGRÁFICAS}

1. Comisión sobre Determinantes Sociales de la Salud. Subsanar las desigualdades en una generación: Alcanzar la equidad sanitaria actuando sobre los determinantes sociales de la salud. Ginebra: Organización Mundial de la Salud; 2008 [Internet]. [citado 1 junio 2012]. Disponible en: http://whqlibdoc.who.int/publications/2009/9789243563701 spa.pdf

2. Commission on Social Determinants of Health. Knowledge Networks. World Health Organisation; 2012 [Internet]. [cited 2012 Jun1]. Available from: http://www.who.int/social determinants/themes/en/ 
3. Muntaner C, Sridharana S, Solar O, Benach J. Against unjust global distribution of power and money: The report of the WHO commission on the social determinants of health: Global inequality and the future of public health policy. J Public Health Policy. 2009;30:16375. doi:10.1057/jphp.2009.15

4. Stevens P. WHO prescription for global poverty. Critical Opinion [Internet]. 2008 [cited 2012 Jun 1]. Available from: http://www.criticalopinion.org/articles/169

5. The Economist. The price of weing well [Internet]. 2008 [cited 2012 Jun 1]. Available from: http://www.economist.com/node/12009974v

6. Fraser Institute. The WHO's sick manifesto. Trade combats inequality and improves health. Fraser Forum 10/08; 2008 [Internet]. [cited 2012 Jun 1]. Available from: http://www.fraserinstitute.org/research-news/search.aspx

7. Stevens P. Bias in WHO Report on the social determinants of health. Lancet. 2009;373:298.

8. Smith DG, Krieger N. Tackling health inequities. BMJ. 2008;337(7669):529.

9. Blakely T. Iconography and Commission on the Social Determinants of Health (and health inequity). J Epidemiol Comm Health. 2008;62(12):1018-20.

10. Berkman LF, Sivaramakrishnan K. WHO Commission on the social determinants of health: a bold new venture. Eur J Public Health. 2008;18(6):298-373.

11. Can health equity become a reality? Lancet. $2008 ; 372: 1607$.

12. López Arellano O, Escudero JC, Carmona LD. Los determinantes sociales de la salud. Una perspectiva desde el Taller Latinoamericano de Determinantes Sociales de la Salud, ALAMES. Medicina Social. 2008;3(4):323-35.

13. Rosenthal S. Engels and the WHO Report. Dissident Voice [Internet]. 2008 [cited 2012 Jun 1]. Available from: http://susanrosenthal.com/articles/engels-and-the-whoreport

14. Cabrera A, Camacho I, Cortés N, Eibenschutz C, González R, Ortiz L, et al. Por una discusión acerca de los informes de la CDSS desde la perspectiva de la medicina social latinoamericana. En: Eibenschutz C, Tamez S, González R, editores.

¿Determinación social o determinantes sociales de la salud? Memoria del Taller Latinoamericano sobre Determinantes Sociales de la Salud. México: Universidad Autónoma Metropolitana; 2011. p. 273-88.

15. Birn AE. Making it Politic(al): Closing the Gap in a Generation: Health Equity through Action on the Social Determinants of Health. Soc Med. 2009;4(3):166-82.

16. Krug EG, Dahlberg LL, Mercy JA, Zwi AB, Lozano R. World report on violence and health. Ginebra: Organización Mundial de la Salud; 2002.

17. Holdstock D. Morbidity and Mortality among Soldiers and Civilians. Taipale I, editor. Londres: Zed Books; 2002.

18. Hobsbawm E. Guerra y Paz en el siglo XXI. Barcelona: Crítica; 2007. 
19. Sidel VW, Onel E, Geiger HF, Leaning J, Foege WH. Public health responses to natural and human-made disasters. En: Last J, Wallace R, Rosenau M, editors. Last public health and preventive medicine. 13a ed. Norwalk, CT: Appleton and Lange; 1992. p. $1173-85$.

20. Borrell C, Muntaner C, Benach J, Artazcoz L. Social class and self-reported health status among men and women: what is the role of work organisation, household material standards and household labour? Soc Sci Med. 2004;58:1869-87.

21. Birn AE. Making it Politic(al): Closing the Gap in a Generation: Health Equity through Action on the Social Determinants of Health. Soc Med. 2009;4(3):166-182.

22. Navarro V. What we mean by social determinants of health. Glob Health Promot. $2009 ; 16(1): 5-16$.

23. Benach J, Muntaner C. La epidemia global de desigualdad en salud tiene su origen en la crisis socio-ecológica del capitalismo. Entrevista. Ecología Política. 2009;37:2133.

24. Muntaner C, Sridharan S, Solar O, Benach J. Against unjust global distribution of power and money: The report of the WHO commission on the social determinants of health: Global inequality and the future of public health policy. J Public Health Policy. $2009 ; 30(2): 16375$.

J oan Benach. Grup de Recerca en Desigualtats en Salut-Employment Conditions Network (GREDS-EMCONET). Departament de Ciències Polítiques i sociología. Universitat Pompeu Fabra. Passeig Circumval-lació, 8 08003. Barcelona, España. Correo electrónico: joan.benach@upf.edu 